\title{
Limited Immunogenicity of Human Induced Pluripotent Stem Cell-Derived Cartilages
}

\author{
Takeshi Kimura, MD, ${ }^{1,2}$ Akihiro Yamashita, DDS, PhD, ${ }^{1}$ Keiichi Ozono, MD, PhD, \\ and Noriyuki Tsumaki, MD, $\mathrm{PhD}^{1}$
}

Articular cartilage damage does not spontaneously heal and could ultimately result in a loss of joint function. Damaged cartilage can be repaired with cell/tissue sources that are transplanted, however, autologous chondrocytes are limited in number as a cell source. Induced pluripotent stem cells (iPSCs) are a relatively new and abundant cell source and can be made from the patient, but at a considerable cost. Because cartilage is immunoprivileged tissue, allogeneic cartilages have been transplanted effectively without matching for human leukocyte antigen (HLA), but are difficult to acquire due to scarcity of donors. In this study, we examined the immunogenicity of human iPSC-derived cartilages (hiPS-Carts) in vitro to evaluate whether allogeneic hiPSCarts can be a new cell/tissue source. The cells in hiPS-Carts expressed limited amounts of major histocompatibility complex (MHC) class I (HLA-ABC) and MHC class II (HLA-DRDQDP). Treatment with interferon $\gamma$ (IFN $\gamma$ ) induced the expression of MHC class I, but not MHC class II in hiPS-Carts. A mixed lymphocyte reaction assay showed that hiPS-Carts stimulated the proliferation of neither T cells nor the activation of NK cells. Furthermore, hiPS-Carts suppressed the proliferation of T cells stimulated with interleukin 2 and phytohemagglutinin (PHA). Together with previously reported findings, these results suggest that hiPS-Carts are no more antigenic than human cartilage. Additionally, in combination with the fact that iPSCs are unlimitedly expandable and thus can supply unlimited amounts of iPS-Carts from even one iPSC line, they suggest that allogeneic hiPS-Carts are a candidate source for transplantation to treat articular cartilage damage.

Keywords: articular cartilage, regeneration, induced pluripotent stem (iPS) cells, allogeneic transplantation

\section{Introduction}

A RTICULAR CARTILAGE COVERS the ends of bones and provides lubrication in the joints. It consists of chondrocytes embedded in the extracellular matrix. Damage to cartilage seldom heals because of limited capacity for intrinsic repair, ultimately resulting in debilitating conditions such as osteoarthritis. Autologous chondrocyte implantation has been widely performed to repair articular cartilage damage, but it is associated with several limitations. First, the chondrocytes must be expanded in vitro, which causes a loss of chondrocytic characteristics and their conversion into fibroblastic cells. The result is the formation of repaired tissue that consists of fibrous tissue. Second, patients are burdened with sacrificing donor sites and two-stage surgery.
The transplantation of allogeneic cartilage, when performed without expansion in culture, resolves these limitations. Cartilage is believed to be immunoprivileged, ${ }^{1,2}$ because of its avascular nature and because chondrocytes are surrounded by the extracellular matrix, which prevents the chondrocytes from being exposed to cells responsible for immunological reactions. In addition, it has been reported that chondrocytes express a limited amount of HLA. ${ }^{2}$ Chondrocytes are even reported to be immunosuppressive. ${ }^{2}$ On the other hand, chondrocytes have been reported to be immunogenic under specific conditions. ${ }^{3}$ Therefore, whether the transplantation of allogeneic cartilage is immunotolerant may depend on the condition of the transplant.

Allogeneic cartilages harvested from juveniles ${ }^{2,4-7}$ have been transplanted to thousands of patients without matching

\footnotetext{
${ }^{1}$ Cell Induction and Regulation Field, Department of Cell Growth and Differentiation, Center for iPS Cell Research and Application, Kyoto University, Kyoto, Japan.

${ }^{2}$ Department of Pediatrics, Osaka University Graduate School of Medicine, Osaka, Japan.

(c) Takeshi Kimura et al., 2016; Published by Mary Ann Liebert, Inc. This Open Access article is distributed under the terms of the Creative Commons Attribution Noncommercial License (http://creativecommons.org/licenses/by-nc/4.0/) which permits any noncommercial use, distribution, and reproduction in any medium, provided the original author(s) and the source are credited.
} 
HLA types or the use of immunosuppressive agents. Cartilages from juveniles have more anabolic activities and are less antigenic than those from adults, ${ }^{2,7}$ but the lack of donors, heterogeneity in the quality of the cartilages obtained from different individuals, and risk of disease transmission suggest that an alternative method is preferred.

Induced pluripotent stem cells (iPSCs) ${ }^{8,9}$ are a potential cell source for all cell types, including chondrocytes. iPSCs are somatic cells, such as skin cells or blood cells that have been reprogrammed to the pluripotent state by the introduction of specific factors. iPSCs have similar properties, such as pluripotency and self-renewal, as embryonic stem cells (ESCs), but do not require the sacrifice of embryos. We previously reported a method to generate scaffoldless cartilage tissues from human iPSCs (hiPSCs). ${ }^{10}$ Three-dimensional culture of chondrogenically differentiated hiPSCs in suspension culture causes these cells to secrete and deposit cartilage extracellular matrix to form cartilage. These hiPSC-derived cartilages (hiPS-Carts) are spherical and about $2 \mathrm{~mm}$ in diameter. When hiPS-Carts were transplanted into defective articular cartilage of mini-pig that was treated with immunosuppressive drugs, they constituted articular cartilage. ${ }^{10}$ These findings suggest that hiPS-Carts can be a new source for transplantation to treat defects of articular cartilage.

Because iPSCs can be made from the patient's own cells, they permit the possibility of autologous transplants. ${ }^{11}$ However, the preparation of patient-iPSCs under good manufacturing practice (GMP) guidelines is costly. Therefore, the purpose of this study is to examine the immunogenicity of hiPS-Carts and discuss the possibility of hiPS-Carts produced from allogeneic hiPSCs for the regeneration of articular cartilage defects.

\section{Materials and Methods}

\section{Ethics statement}

All experiments were approved by the institutional review board, institutional animal committee (as appropriate), and the institutional biosafety committee of Kyoto University, and the institutional review board of the University of Toyama and Hachiya Orthopedic Hospital.

\section{Cells and tissues}

A description of the cells/tissues used in this study is provided in Figure 1A. All abbreviations used in this article are listed in Table 1.

Human iPSCs. Two iPSC lines, 409B2 and 604B1, generated from different healthy individuals, were gifts from K. Okita and S. Yamanaka (Center for iPS Cell Research and Application [CiRA], Kyoto University, Kyoto, Japan). ${ }^{12}$ 409B2 was generated from human dermal fibroblasts, and 604B1 from human peripheral mononuclear cells. Episomal plasmid vectors (pCXLE-hOCT3/4-shp53-F, hSK, hUL, EBNA1) were electroporated, and all cells were negative for genome integration.

hiPSC-derived cartilages. hiPSCs were chondrogenically differentiated to produce hiPS-Carts for 8 weeks as described previously. ${ }^{10}$ Briefly, the hiPSCs were transferred and then maintained in a feeder-free medium, Essential 8 (Thermo Fisher

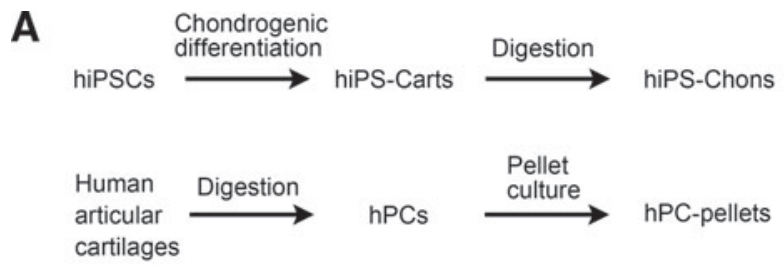

hPBMCs (purchased)

hMVECs (purchased)
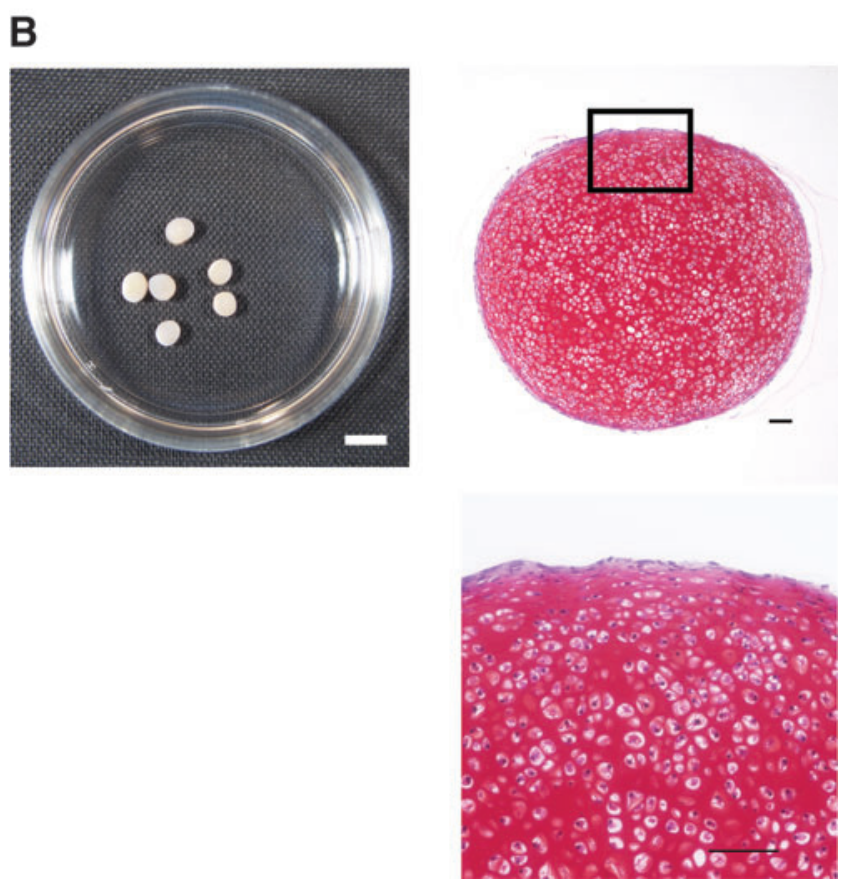

FIG. 1. Cells/tissues used in this study. (A) Different cell sources are shown. (B) hiPS-Carts. Left, gross appearance of hiPS-Carts. Right top, histology of a hiPS-Cart. The boxed region in the right top panel is shown in the right bottom. Safranin O-Fast Green-Iron Hematoxylin staining. Scale bars: left top, $5 \mathrm{~mm}$; right top and right bottom, $100 \mu \mathrm{m}$. hiPSCs, human induced pluripotent stem cells; hiPS-Carts, hiPSCderived cartilages; hiPS-Chons, chondrocytes isolated from hiPS-Carts; hMVECs, human microvascular endothelial cells; hPBMCs, human peripheral blood mononuclear cells; hPCs, human primary chondrocytes; hPC pellets, cultured pellets prepared from hPCs.

Scientific), with $50 \mathrm{U} / \mathrm{mL}$ penicillin and $50 \mathrm{mg} / \mathrm{mL}$ streptomycin $(\mathrm{P} / \mathrm{S})$ (Thermo Fisher Scientific) in 3.5-cm Matrigel-coated dishes. The hiPSCs formed high-density cell colonies consisting of $1-2 \times 10^{5}$ cells $10-15$ days after the start of maintenance under feeder-free culture conditions. Subsequently, chondrogenic differentiation of the iPSCs was performed. The hiPSCs were initially differentiated into mesendodermal cells in Dulbecco's modified Eagle's medium (DMEM)/F12 (Sigma) with $10 \mathrm{ng} / \mathrm{mL}$ of Wnt3A (R\&D), $10 \mathrm{ng} / \mathrm{mL}$ of Activin A (R\&D), $1 \%$ ITS (Thermo Fisher Scientific), $1 \%$ fetal bovine serum (FBS), and P/S for 3 days. On day 3, the medium was changed to basal medium (DMEM [Sigma], with $1 \%$ ITS, $1 \%$ FBS, $2 \mathrm{mM}$ L-glutamine [Thermo Fisher Scientific], $1 \times 10^{-4}$ $\mathrm{M}$ nonessential amino acids [Thermo Fisher Scientific], $1 \mathrm{mM} \mathrm{Na}$ pyruvate [Thermo Fisher Scientific], and P/S) 
Table 1. Abbreviations Used in the Article

\begin{tabular}{ll}
\hline Abbreviations & \multicolumn{1}{c}{ Full spelling } \\
\hline iPSC & Induced pluripotent stem cell \\
HLA & Human leukocyte antigen \\
hiPS-Cart & Human iPSC-derived cartilage \\
MHC & Major histocompatibility complex \\
IFN $\gamma$ & Interferon $\gamma$ \\
IL2 & Interleukin 2 \\
PHA & Phytohemagglutinin \\
ESC & Embryonic stem cell \\
GMP & Good manufacturing practice \\
hiPS-Chon & Human iPSC-derived chondrocyte \\
Hpc & Human primary chondrocyte \\
hPC-pellet & hPC-derived pellet \\
hPBMC & Human peripheral blood mononuclear cell \\
hMVEC & Human microvascular endothelial cell \\
CFSE & Carboxyfluorescein diacetate \\
& $\quad$ succinimidyl ester \\
BrdU & Bromodeoxyuridine \\
APC & Antigen-presenting cell \\
\hline
\end{tabular}

supplemented with $50 \mu \mathrm{g} / \mathrm{mL}$ of ascorbic acid (Nacalai), $10 \mathrm{ng} / \mathrm{mL}$ of BMP2 (PeproTech), $10 \mathrm{ng} / \mathrm{mL}$ of TGF $\beta 1$ (PeproTech) and $10 \mathrm{ng} / \mathrm{mL}$ of GDF5 (PTT), which was intended to commit the cells to the chondrocytic lineage. A total of $10 \mathrm{ng} / \mathrm{mL}$ of bFGF (WAKO) was added to the medium from day 3 to 14 . Multilayered nodules were formed by day 14, and the nodules were physically separated from the bottom of the dishes to form particles, which were then transferred to a suspension culture in 3.5-cm Petri dishes on day 14. The cells in the particles produce cartilaginous extracellular matrix, resulting in the particles becoming cartilaginous tissue in suspension culture. The culture medium was changed every 2-7 days. The hiPSC-derived cartilaginous particles on day 56 were used for the study. The appearance and histology of hiPS-Carts are shown in Figure 1B. One iPS-Cart contains around $7.1 \times 10^{4}$ cells. $^{10}$

Chondrocytes in hiPS-Carts. Chondrocytes isolated from hiPS-Cart (hiPS-Chons) were prepared by enzymatically digesting hiPS-Carts. The hiPS-Carts were treated with $0.25 \%$ trypsin-EDTA (Thermo Fisher Scientific) for $1 \mathrm{~h}$ and subsequently with $4 \mathrm{mg} / \mathrm{dL}$ Collagenase D (Roche Diagnostics GmbH, Mannheim, Germany) in DMEM supplemented with $2 \mathrm{mM}$ L-glutamine and P/S overnight. After washing, hiPS-Chons were subjected to flow cytometry analysis or suspended in DMEM supplemented with $10 \%$ FBS, $1 \mathrm{mM}$ sodium pyruvate, $1 \%$ ITS-X, $10 \mathrm{ng} / \mathrm{mL}$ TGF1 $\beta$ $1,50 \mu \mathrm{g} / \mathrm{mL}$ ascorbic acid, $100 \mathrm{nM}$ dexamethasone and $\mathrm{P} / \mathrm{S}$ (chondrogenic medium), and plated for subsequent treatment with mitomycin C (Kyowa Hakko).

Human primary chondrocytes. Human primary chondrocytes (hPCs) were obtained from surgical specimens discarded after total knee joint arthroplasty surgery. Articular cartilage from less damaged areas was harvested from the distal femur of five different individuals. The cartilage was chipped and treated with $0.25 \%$ trypsin-EDTA for $1 \mathrm{~h}$ and $4 \mathrm{mg} / \mathrm{dL}$ Collagenase D overnight to isolate hPCs as described previously. ${ }^{13}$ Then, hPCs were plated on $10-\mathrm{cm}$ dishes and cultured in chondrogenic medium for 7 days.
hPC pellets. In a $15-\mathrm{mL}$ conical tube (Corning), $5 \times 10^{5}$ hPCs were centrifuged ( $300 \mathrm{~g}, 5 \mathrm{~min})$ to form a pellet. The pellets were cultured in a chondrogenic medium for 4 weeks as described previously ${ }^{13}$ to form $\mathrm{hPC}$ pellets.

Human peripheral blood mononuclear cells. Human peripheral blood mononuclear cells (hPBMCs) were purchased (lot No. 0000363787/0000441878; Lonza, Basel, Switzerland). Cryopreserved hPBMCs were thawed and suspended in RPMI1640 (Thermo Fisher Scientific) supplemented with $10 \% \mathrm{FBS}, \mathrm{P} / \mathrm{S}$.

Human microvascular endothelial cells. Human microvascular endothelial cells (hMVECs) were purchased (lot No. 000412510; Lonza) and used as control cells with high immunogenicity. ${ }^{14}$ Cryopreserved hMVECs were thawed, plated in the Endothelial Cell Growth Medium-2 BulletKit ${ }^{\mathrm{TM}}$ (Lonza) and subjected to treatment with mitomycin C.

\section{Determination of HLA types}

HLA typing was performed with Luminex 200 system (Luminex) and the WAKFlow HLA Typing Kit (Wakunaga, Hiroshima, Japan) at the HLA Laboratory (Kyoto, Japan). ${ }^{15}$

\section{Flow cytometry analysis}

We analyzed the expression of cell surface markers by flow cytometry using FACS Aria II flow cytometer (BD Biosciences) and FlowJo version 7.6.5 software (FlowJo). hiPS-Chons, hPCs, and hPBMCs were stained with antihuman antibodies at $4^{\circ} \mathrm{C}$ for $30 \mathrm{~min}$. The antibodies used are described in Table 2. HLA expression was also analyzed after treating the cells with $25 \mathrm{ng} / \mathrm{mL}$ interferon $\gamma$ (IFN $\gamma$ ) (Sigma) for $72 \mathrm{~h}$. Isotype control antibodies were used (Table 2). We also, respectively, analyzed $\mathrm{T}$ cell proliferation and regulatory $\mathrm{T}$ cell population with flow cytometer using the CellTrace ${ }^{\mathrm{TM}}$ Carboxyfluorescein Diacetate Succinimidyl Ester (CFSE) Cell Proliferation Kit (Thermo Fisher Scientific) and the Human FOXP3 Staining Kit (BD Pharmingen $^{\text {TM }}$ ) as described below.

\section{Messenger RNA expression analysis}

hiPS-Carts were frozen in liquid nitrogen and crushed by Multi-Beads Shocker (Yasui Kikai), and total RNA was extracted using TRIzol ${ }^{\circledR}$ (Thermo Fisher Scientific). Total RNAs were also extracted from hPCs and hPBMCs. For realtime quantitative reverse transcription PCR (qRT-PCR), $1 \mu \mathrm{g}$ of total RNA was reverse transcribed into first-strand complementary DNA (cDNA) by using ReverTra Ace (Toyobo, Tokyo, Japan) and an oligo(dT)20 primer. The polymerase chain reaction (PCR) amplification was performed using the KAPA SYBR FAST qPCR Kit Master Mix ABI prism (KAPA Biosystems). The PCR primers used are listed in Table 3. The RNA expression levels were normalized to the level of GAPDH expression. Results indicate the relative expression of the molecules $\left({ }^{\Delta \Delta} \mathrm{Ct}\right.$ : control cells $\left.=1\right)$.

\section{Pretreatment of stimulator cells with mitomycin $C$ before coculture}

Two sets of stimulator cells, $1-5 \times 10^{6}$ hiPS-Chons and 1.6- $2.4 \times 10^{6}$ hMVECs, were pretreated with $10 \mu \mathrm{g} / \mathrm{mL}$ 
Table 2. Antibodies Used for Flow Cytometry

\begin{tabular}{lllcc}
\hline Antigen & \multicolumn{1}{c}{ Clone } & Isotype & Fluorochrome & Company \\
\hline CD3 & UCHT1 & Mouse IgG1, $\kappa$ & APC & BD Pharmingen \\
CD4 & RPA-T4 & Mouse IgG1, $\kappa$ & APC & BD Pharmingen \\
CD8 & RPA-T8 & Mouse IgG1, $\kappa$ & PE & BD Pharmingen \\
CD25 & M-A251 & Mouse IgG1, $\kappa$ & FITC & BD Pharmingen \\
CD56 & B159 & Mouse IgG1, $\kappa$ & PE & BD Pharmingen \\
CD69 & FN50 & Mouse IgG1, $\kappa$ & FITC & BD Pharmingen \\
CD80 & L307.4 & Mouse IgG1, $\kappa$ & FITC & BD Pharmingen \\
CD86 & $2331(F U N-1)$ & Mouse IgG1, $\kappa$ & APC & BD Pharmingen \\
CD273 & MIH18 & Mouse IgG1, $\kappa$ & APC & BD Pharmingen \\
CD274 & MIH1 & Mouse IgG1, $\kappa$ & FITC & BD Pharmingen \\
HLA-ABC & G46-2.6 & Mouse IgG1, $\kappa$ & APC & BD Pharmingen \\
HLA-DR, DP.DQ & Tu39 & Mouse IgG2a, $\kappa$ & FITC & BD Pharmingen \\
FoxP3 & $259 D / C 7$ & Mouse IgG1, $\kappa$ & PE & BD Pharmingen \\
Isotype control & MOPC-21 & Mouse IgG1, $\kappa$ & APC & BD Pharmingen \\
& & & FITC & BD Pharmingen \\
& & & PE & BD Pharmingen \\
& & Mouse IgG2a, $\kappa$ & FITC & BD Pharmingen \\
\hline
\end{tabular}

APC, antigen-presenting cell; HLA, human leukocyte antigen.

mitomycin $\mathrm{C}$ in 100-mm dishes for $3 \mathrm{~h}$ to arrest cell division before coculture. hiPS-Carts and hPC pellets were not subjected to this treatment.

\section{Cell proliferation analysis after coculture}

T cell proliferation was analyzed by the CellTrace CFSE Cell Proliferation Kit (Thermo Fisher Scientific). Briefly, hPBMCs were pretreated with CFSE before the start of the coculture. The number of divisions by T-cells, which were indicated by a high expression level of CD4, was detected by flow cytometry analysis after coculture. The proliferation rates of hPBMCs after coculture were analyzed by the Bromodeoxyuridine (BrdU) Cell Proliferation ELISA Kit (Abcam, Cambridge, United Kingdom). BrdU was added $8 \mathrm{~h}$ before the end of coculture. Colorimetric detection of BrdU incorporation was performed with the Envision multilabel plate reader (PerkinElmer).

\section{Mixed lymphocyte assay}

$2 \times 10^{5} \mathrm{hPBMCs}$ were cocultured with one hiPS-Cart, one hPC pellet, or $1 \times 10^{5}$ mitomycin C-treated hMVECs in RPMI1640 supplemented with $10 \%$ FBS and P/S in one well of a 96-well plate for $96 \mathrm{~h}$ and subjected to the following analysis.

Table 3. The Sequence of Primers USED FOR QRT-PCR

\begin{tabular}{lc}
\hline Primer & \multicolumn{1}{c}{ Sequence } \\
\hline GAPDH $\mathrm{F}$ & ACCCAGAAGACTGTGGATGG \\
GAPDH R & TTCTAGACGGCAGGTCAGGT \\
$H L A-A \mathrm{~F}$ & GCGGCTACTACAACCAGAGC \\
$H L A-A \mathrm{R}$ & CCAGGTAGGCTCTCAACTGC \\
$H L A-B \mathrm{~F}$ & TCCTAGCAGTTGTGGTCATG \\
$H L A-B \mathrm{R}$ & TCAAGCTGTGAGAGACACAT \\
$H L A-C \mathrm{~F}$ & TCCTGGTTGTCCTAGCTGTC \\
$H L A-C \mathrm{R}$ & CAGGCTTTACAAGTGATGAG
\end{tabular}

qRT-PCR, real-time quantitative reverse transcription PCR.
The proliferations of $\mathrm{CD}^{+} \mathrm{T}$ cells and $\mathrm{hPBMCs}$ were, respectively, analyzed by the CFSE Kit and the BrdU Kit as described above.

hiPS-Carts and hPC pellets after coculture were fixed with 4\% paraformaldehyde, embedded in paraffin, and subjected to preparation for histological sections. Semi-serial sections were stained with Safranin O-Fast Green-Iron Hematoxylin and immunostained with an anti-HLA-ABC antibody (ab134189, 1:200; Abcam). Secondary antibodies conjugated to Alexa Fluor 594 (1:1000; Thermo Fisher Scientific,). DAPI (1:1000; Dojindo Molecular Technologies, Kumamoto, Japan) was used to detect immune complexes.

For analysis of the NK cell response, hPBMCs after coculture with hiPS-Carts or hPC pellets for $4 \mathrm{~h}$ were subjected to flow cytometry analysis to measure the expression of CD69 and CD56. hPBMCs stimulated with $5 \mathrm{ng} / \mathrm{mL}$ interleukin 2 (IL2; Sigma) for $4 \mathrm{~h}$ were used as control.

\section{Immunosuppression assay}

$2 \times 10^{5}$ hPBMCs were cocultured with one hiPS-Cart or $1 \times 10^{5}$ mitomycin C-treated hiPS-Chons in the presence of $5 \mathrm{ng} / \mathrm{mL}$ IL2 and 1\% PHA-M for $96 \mathrm{~h}$. The proliferation of $\mathrm{CD} 4^{+} \mathrm{T}$ cells was detected by flow cytometry analysis using the CFSE Kit. CFSE was added before coculture. hPBMCs cultured in the absence of hiPS-Carts or hiPS-Chons were used as control.

$2 \times 10^{5} \mathrm{hPBMCs}$ were cocultured with one hiPS-Cart in the absence of $5 \mathrm{ng} / \mathrm{mL}$ IL2 and $1 \%$ PHA-M for $96 \mathrm{~h}$. The populations of regulatory $\mathrm{T}$ cells were measured by flow cytometry analysis using anti-CD4 and anti-CD25 antibodies and the Human FOXP3 Staining Kit (BD Pharmingen).

\section{Immunosuppression assays by coculture using culture inserts or culture in the conditioned media}

To examine whether secreted factors from hiPS-Carts mediate immunosuppressive activities, hPBMCs were cocultured using a cell culture insert or cultured in the conditioned medium in the presence of $5 \mathrm{ng} / \mathrm{mL}$ IL2 and $1 \%$ 
Table 4. Human Leukocyte Antigen Alleles of Cells

\begin{tabular}{|c|c|c|c|c|c|c|c|c|}
\hline \multirow[b]{2}{*}{ hiPSC line, 409B2 } & \multicolumn{2}{|c|}{$H L A-A$} & \multicolumn{2}{|c|}{$H L A-B$} & \multicolumn{2}{|c|}{$H L A-C w$} & \multicolumn{2}{|c|}{$H L A-D R B 1$} \\
\hline & A2 & & B27 & B50 & $\mathrm{Cw} 1$ & $\mathrm{Cw} 4$ & DR1 & DR13 \\
\hline hiPSC line, 604B1 & $\mathrm{A} 1$ & A24 & B7 & B37 & Cw6 & Cw7 & DR1 & DR9 \\
\hline hMVEC & A1 & A2 & B62 & B3901 & Cw10 & Cw5 & DR4 & DR8 \\
\hline hPBMC-1 & A3 & & B7 & & Cw7 & & DR8 & DR15 \\
\hline hPBMC-2 & A2 & A68 & B7 & B60 & Cw10 & $\mathrm{Cw} 7$ & DR6 & DR4 \\
\hline
\end{tabular}

hiPSC, human iPSC; hMVEC, human microvascular endothelial cell; hPBMC, human peripheral blood mononuclear cell.

PHA-M. After that, the proliferation of $\mathrm{CD}^{+} \mathrm{T}$ cells was detected by flow cytometry analysis using the CFSE Kit.

$4 \times 10^{5}$ hPBMCs were cocultured with or without two hiPS-Carts separated in the Millicell ${ }^{\circledR}$ cell culture insert (Merck, Darmstadt, Germany) in RPMI1640 supplemented with $10 \% \mathrm{FBS}$ and P/S in one well of a 24-well plate for $96 \mathrm{~h}$.

We prepared the conditioned media by culturing two hiPS-Carts in RPMI1640 supplemented with 10\% FBS and $\mathrm{P} / \mathrm{S}$ in one well of the 24 -well plate for $24 \mathrm{~h}$. The $2 \times 10^{5}-$ stimulated hPBMCs were cultured in conditioned media in one well of the 96-well plate for $96 \mathrm{~h}$. The hPBMCs cultured in RPMI1640 supplemented with 10\% FBS and P/S were used as control.

\section{Statistical analysis}

Data are shown as averages and standard deviations. In this study, we used two-tailed Student's $t$-tests or one-way analysis of variance with Tukey's HSD tests for multiple comparisons. $p$-values, 0.05 were considered to be statistically significant.

\section{Results}

\section{HLA types of cells used in this study}

Human peripheral blood mononuclear cells (hPBMCs) from two different individuals (hPBMCs-1 and -2), hiPSCs
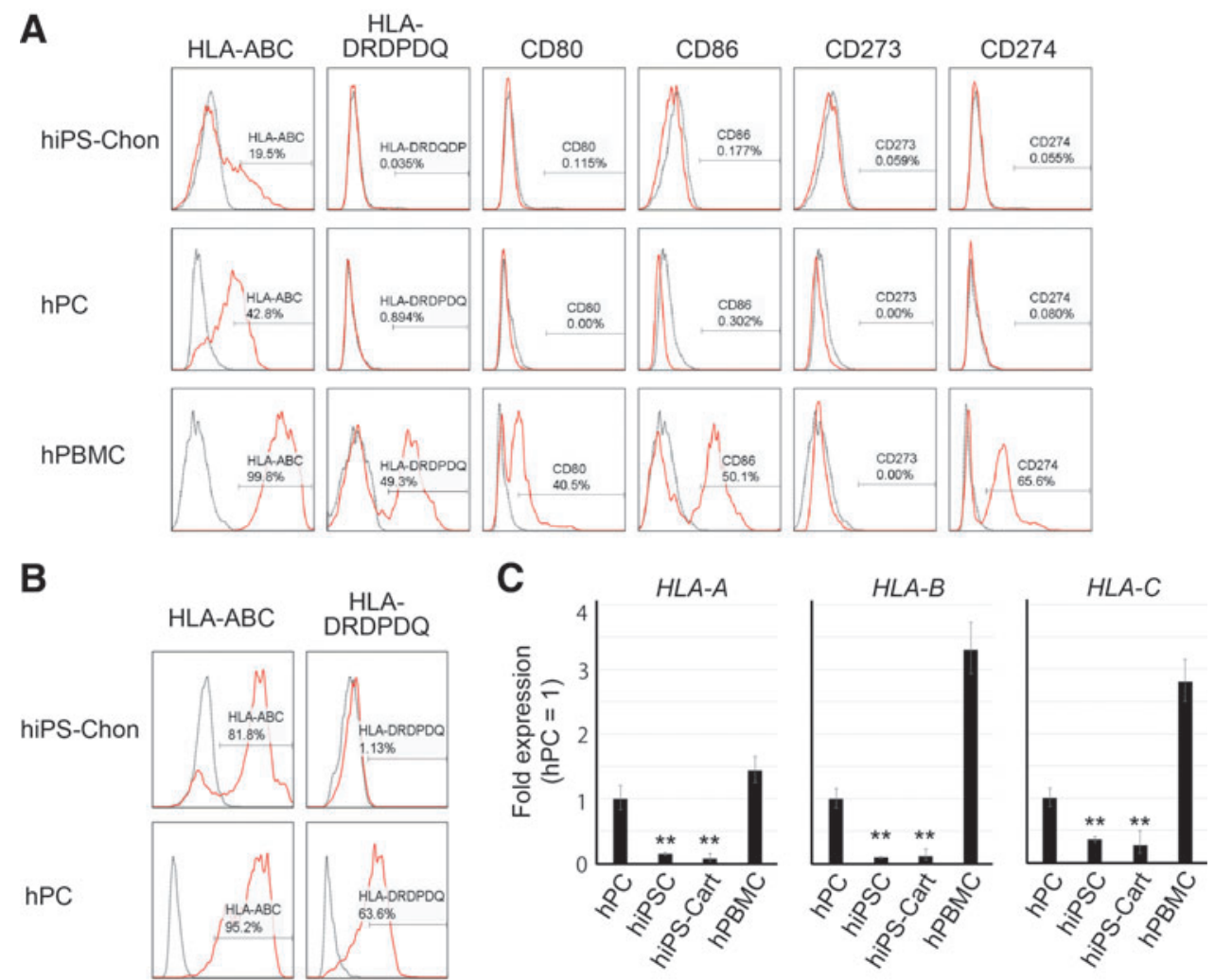

FIG. 2. Expression analysis of HLA and costimulatory molecules in hiPS-Chons. (A, B) Flow cytometry analysis of the expression of cell surface markers in hiPS-Chons and hPCs without (A) or with (B) INF $\gamma$ stimulation using antibodies, which recognize the indicated proteins (red lines). Black dotted lines represent data from the isotype control. Data are representative of independent experiments using hiPS-Chons from iPSCs from two different individuals (409B2 and 604B1) and hPCs from three different individuals. (C) Real-time quantitative reverse transcription PCR (qRT-PCR) analysis of the expression of $H L A-A, H L A-$ $B$, and $H L A-C$. Error bars denote mean \pm SD. $n=3$ technical replicates. $* * p<0.01$ versus hPC by Tukey's HSD test. Data are representative of three independent experiments using 409B2 iPSCs. HLA, human leukocyte antigen; iPSCs, induced pluripotent stem cells; SD, standard deviation. 
from two different individuals (409B2 and 604B1), hMVECs from one individual, and hPCs from five different individuals were used in this study. An analysis of HLA types showed that each hPBMC, hiPSC, and hMVEC had unique HLA types and that any combination of hPBMCs and hiPSCs or hMVECs resulted in a mismatch for HLA (Table 4). The HLA types of hPCs were not analyzed.

\section{Analysis of HLA and costimulatory molecules in hiPS-Chons}

hiPS-Carts have features of hyaline cartilage, that is, chondrocytes embedded in cartilage extracellular matrix. ${ }^{10} \mathrm{We}$ isolated chondrocytes from hiPS-Carts (hiPS-Chons) by digesting
hiPS-Carts with collagenase to release the chondrocytes from their surrounding matrix (Fig. 1). hiPS-Chons were subjected to flow cytometry to analyze the expression of cell surface markers. hPCs are known to express limited amounts of major histocompatibility complex (MHC), whereas PBMCs express substantial MHC. We used these two cell types as the control.

hiPS-Chons expressed a limited amount of MHC class I (HLA-ABC) and little MHC class II (HLA-DRDQDP) and costimulatory molecules, B7-1 (CD80), B7-2 (CD86), B7DC (CD273), and B7-H1 (CD274) (Fig. 2A). Treatment with IFN $\gamma$ stimulated the expression of HLA-ABC in hiPSChons (Fig. 2B). hPCs, which were recovered from the less damaged area of articular cartilage from individuals with osteoarthritis, showed HLA-ABC expression even without

A

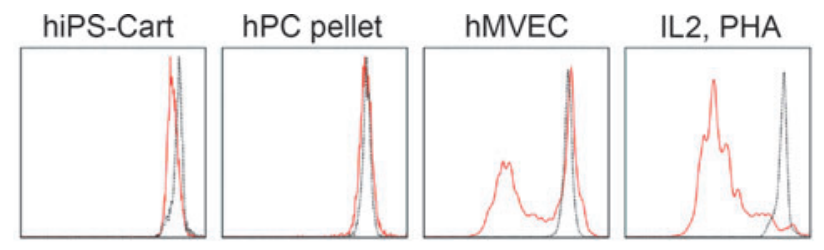

CFSE

B

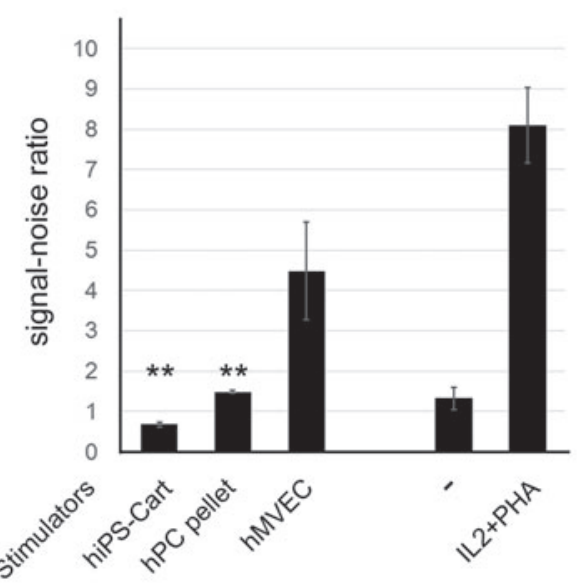

C
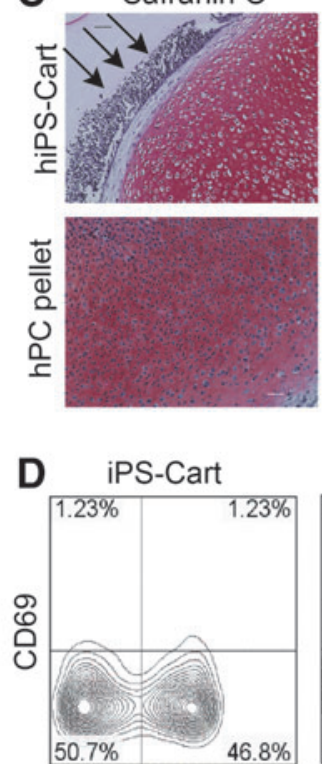
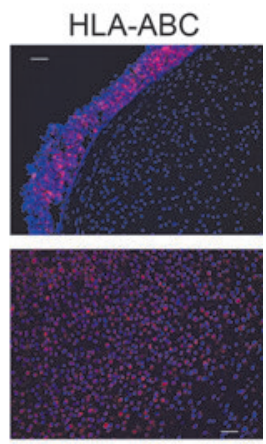

IL2

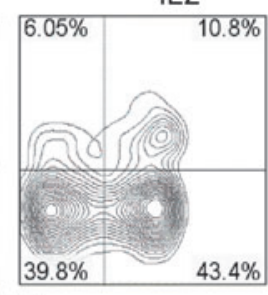

CD56

FIG. 3. Mixed lymphocyte reaction assay. hPBMCs were cocultured with stimulators, such as hiPS-Carts, hPC-pellets, or mitomycin C-treated hMVECs, or cultured in the presence of $5 \mathrm{ng} / \mathrm{mL}$ IL2 and 1\% PHA for $96 \mathrm{~h}$. (A) After coculture, the hPBMCs were subjected to flow cytometry analysis for CD4 and CFSE to assess the number of cell divisions by CD4 ${ }^{+} \mathrm{T}^{2}$ cells. Black dotted lines represent data from hPBMCs cultured in the absence of stimulator cells or IL2/PHA. Data are representative of independent experiments using hPBMCs from two different individuals in combination with hiPS-Carts from two different individuals or with hPC pellets from two different individuals. (B) BrdU was added $8 \mathrm{~h}$ before the end of the coculture or culture. The amounts of incorporated BrdU were measured using a plate reader. Error bars denote mean \pm SD. $n=3$ wells. ${ }^{*} p<0.01$ versus hMVEC by Tukey's HSD test. Data are representative of independent experiments using hPBMCs from two different individuals in combination with hiPS-Carts from two different individuals or with hPC pellets from two different individuals. (C) Histological analysis of hiPS-Carts and hPC pellets after coculture. Semiserial sections were stained with Safranin O-Fast Green-Iron Hematoxylin or immunostained with anti-HLA-ABC antibody (red). Blue color is DAPI. Arrows indicate hPBMCs. Scale bars: $50 \mu \mathrm{m}$. The images are representative of independent experiments using hPBMCs from two different individuals in combination with hiPS-Carts from two different individuals. (D) After coculture, cells were collected and subjected to flow cytometry analysis for CD56 and CD69. hPBMCs stimulated with $5 \mathrm{ng} / \mathrm{mL}$ IL2 for $4 \mathrm{~h}$ were used as controls. Data are representative of two independent experiments using hiPS-Carts from two different individuals. BrdU. Bromodeoxyuridine; CFSE, carboxyfluorescein diacetate succinimidyl ester; hPCpellet, hPC-derived pellet; IL2, interleukin 2; PHA, phytohemagglutinin. 
IFN $\gamma$ stimulation. Treatment with IFN $\gamma$ stimulated the expression of HLA-DRDQDP in hPCs, but not in hiPS-Chons.

Real-time RT-PCR expression analysis showed that the messenger RNA (mRNA) expression levels of $H L A-A, H L A$ $B$, and $H L A-C$ were lower in hiPS-Carts than in hPCs or hPBMCs (Fig. 2C).

\section{Mixed lymphocyte reaction assay}

Although the expression of MHC antigens in hiPSC-Chons is limited, these cells elicit immunological reactions. Like cartilage, hiPS-Carts are avascular and contain chondrocytes surrounded by extracellular matrix, preventing chondrocytes from exposure to blood cells. hiPS-Carts should therefore be less immunogenic than hiPS-Chons, which is one of the reasons why we plan to transplant hiPS-Carts, but not hiPS-Chons in future clinical settings. Accordingly, we examined the immunogenicity of hiPS-Carts. We performed mixed lymphocyte reaction assays by coculturing hPBMCs with hiPS-Carts, hPC-derived pellets (hPC-pellets) or hMVECs. hMVECs are known to stimulate the proliferation of hPBMCs and therefore were used
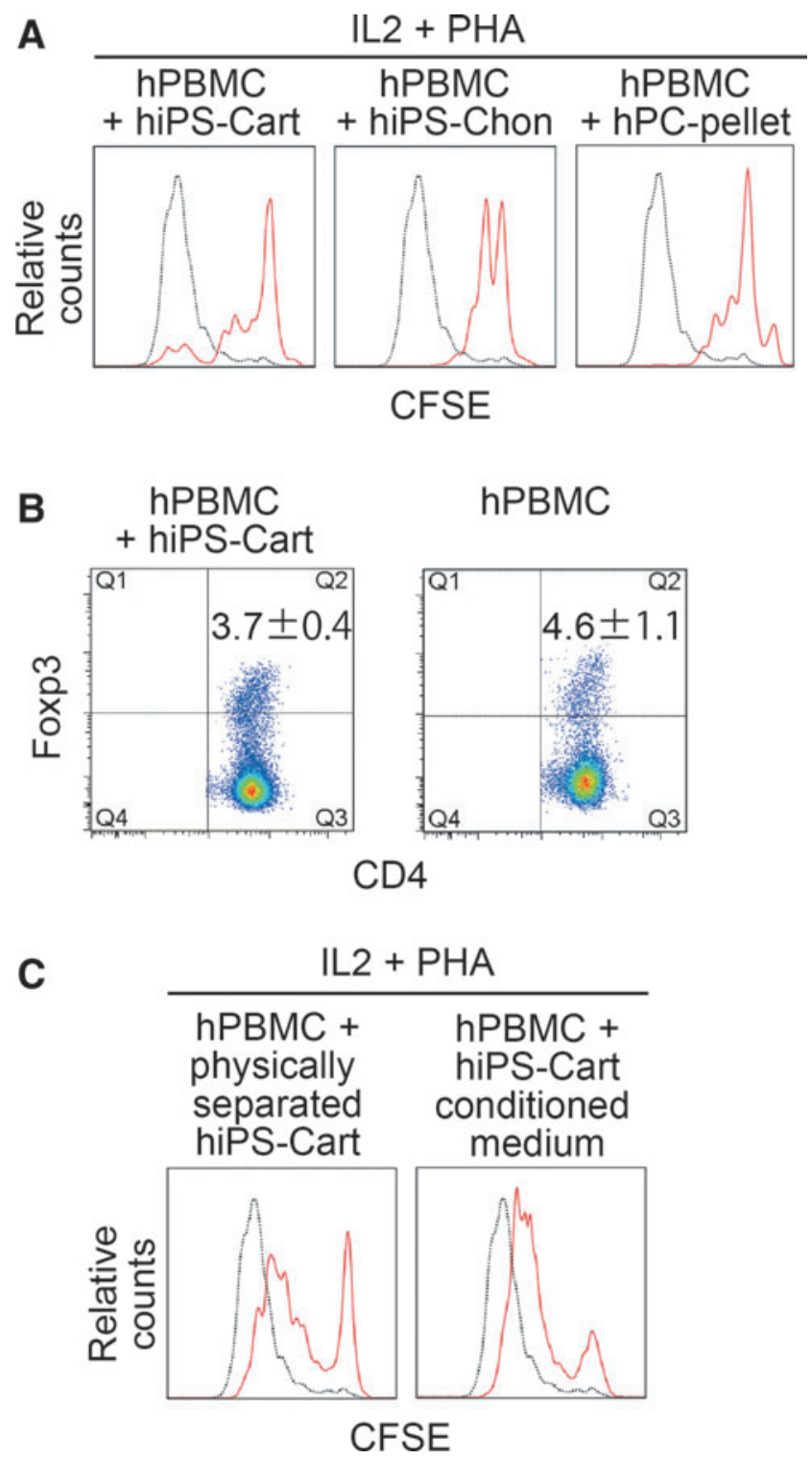

as controls. An analysis of proliferation using CFSE showed that hiPS-Carts and hPC-pellets did not stimulate the proliferation of CD4-positive T lymphocytes, whereas hMVECs did (Fig. 3A). Analysis by BrdU labeling showed that the proliferation rates of hPBMCs were much lower in coculture with hiPS-Carts or $\mathrm{hPC}$ pellets than in coculture with hMVECs (Fig. 3B). Immunohistochemical analysis after coculture with hPBMCs showed that cells in hiPS-Cart did not express HLA-ABC, but chondrocytes in the hPC pellet did (Fig. 3C). These results indicate that hiPS-Carts have limited immunogenicity.

Cells without MHC class I molecules (HLA-ABC) are detected and rejected by the innate immune system. We, therefore, examined the response of NK cells in hPBMCs to hiPS-Carts (Fig. 3D). As a control, we induced activated NK cells $\left(\mathrm{CD}^{+} 9^{+} \mathrm{CD}^{+} 6^{+}\right)$in the NK cells $\left(\mathrm{CD}^{+} 6^{+}\right)$by treatment with IL2. hiPS-Carts did not induce activated NK cells $\left(\mathrm{CD} 9^{+} \mathrm{CD} 6^{+}\right)$, suggesting that hiPS-Carts do not elicit the innate immune response.

\section{Immunosuppressive activities of hiPS-Carts and hiPS-Chons}

We performed coculture of hPBMCs activated by IL 2 and PHA with hiPS-Carts, hiPS-Chons, and hPC-derived pellets (hPC-pellets). An analysis of the proliferation using CFSE showed that, like hPC-pellets, hiPS-Carts and hiPS-Chons suppressed the proliferation of CD4-positive T cells (Fig. 4A). One hiPS-Cart tended to suppress the proliferation of $\mathrm{T}$ lymphocytes more than did $1 \times 10^{5}$ hiPS-Chons.

We then tried to gain some insights into the mechanism responsible for the suppression of $\mathrm{T}$ cell proliferation by hiPS-Carts. Immunosuppressive effects are known to be induced by regulatory $\mathrm{T}$ cells. hiPS-Carts, however, did not

FIG. 4. Immunosuppressive activities of hiPS-Carts and hiPS-Chons. (A) hPBMCs were cocultured with (red lines) or without (black dotted lines) one hiPS-Cart, mitomycin Ctreated $1 \times 10^{5}$ hiPS-Chons, or one hPC-pellet in the presence of IL2 and PHA in one well of a 96-well plate for $96 \mathrm{~h}$. After coculture, cells were subjected to flow cytometry analysis for CD4 and CFSE to assess the number of cell divisions by CD $4^{+}$ $\mathrm{T}$ cells. Data are representative of independent experiments using hPBMCs from two different individuals in combination with hiPS-Carts from two different individuals, with hiPSChons from two different individuals or with hPC-pellets from two different individuals. (B) After coculture with (left) or without (right) one hiPS-Cart in the absence of IL2 and PHA, $\mathrm{hPBMCs}$ were subjected to flow cytometry analysis using antiCD4 and anti-CD25 antibodies and the Human FOXP3 Staining Kit (BD Pharmingen ${ }^{\mathrm{TM}}$ ) to detect the population of regulatory T cells. Error bars denote mean \pm SD. $n=4$. Data are representative of independent experiments using hPBMCs from two different individuals in combination with hiPS-Carts from two different individuals. (C) Analysis of the immunosuppressive activities of secreted factors from hiPS-Carts. The proliferation of $\mathrm{CD}^{+}{ }^{+} \mathrm{T}$ cells was detected by flow cytometry analysis using the CFSE Kit after culture. Data are representative of two independent experiments using hiPS-Carts from two different individuals. Left, hPBMCs were cocultured with (red line) or without (black dotted line) hiPS-Carts physically separated by a transwell insert in the presence of IL 2 and PHA. Right, hPBMCs were cultured in the conditioned medium of hiPS-Carts (red line) or in fresh medium (black dotted line) in the presence of IL2 and PHA. 
increase the population of regulatory $\mathrm{T}$ cells, as indicated by $\mathrm{CD} 25^{+} \mathrm{CD}^{+} \mathrm{FOXP}^{+}$expression (Fig. 4B), suggesting that the suppression of $\mathrm{T}$ cell proliferation by hiPS-Carts is not mediated by regulatory $\mathrm{T}$ cells.

The immunosuppressive property of hiPS-Carts was further assessed by physically separating these cells from activated $\mathrm{CD}^{+} \mathrm{T}$ cells using transwell inserts. These inserts allow the passage of soluble mediators between two chambers, but prevent direct cell-cell contact. Under these conditions, hiPSCarts did not suppress the proliferation of T cells in hPBMCs (Fig. 4C, left). In a separate experiment, the addition of conditioned media obtained by culturing hiPS-Carts to the culture of stimulated hPBMCs did not suppress the proliferation of $\mathrm{T}$ cells in hPBMCs (Fig. 4C, right). These results suggest that cell-cell contact may be necessary for the immunosuppressive property of hiPS-Carts.

\section{Discussion}

The immune rejection of allogeneic transplanted tissue is elicited when the recipient's $\mathrm{T}$ lymphocytes recognize the transplanted tissue as "nonself". ${ }^{16}$ This rejection occurs through several mechanisms. One mechanism is activated by direct contact with the intact MHC class I located on the cell surface of the transplanted allogeneic cells, which activates the recipient's T cells. In the present article, flow cytometry analysis showed that hiPS-Chons expressed a limited amount of MHC class I, suggesting that allogeneic hiPS-Carts would not elicit this mechanism robustly. Another mechanism is the presentation of proteins such as MHC from the transplanted allogeneic tissues by the recipient's antigen-presenting cells (APC), thus stimulating helper $\mathrm{T}$ cells and subsequently $\mathrm{B}$ cells to produce antibodies and macrophages. Again, however, a limited expression of MHC class I and class II in hiPS-Chons suggests that this mechanism was not activated robustly.

IFN $\gamma$ is known to stimulate the expression of MHC. Treatment with IFN $\gamma$ did not stimulate the expression of HLA-DRDQDP in hiPS-Chons, but did in hPCs. The hPCs in this study were from aged individuals with osteoarthritis. It has been reported that chondrocytes from juvenile donors are less antigenic than chondrocytes from adult donors, because treatment with IFN $\gamma$ stimulates the expression of HLA-DR only in the chondrocytes from adult donors. ${ }^{2}$ These lines of observations suggest that hiPS-Chons are more similar to chondrocytes from juvenile donors than to those from adult donors. This finding is consistent with the previous finding that hiPS-Carts, when implanted in the subcutaneous spaces of SCID mice, initiate embryonic skeletal development, ${ }^{10}$ suggesting that hiPS-Carts correspond to primordial cartilage in embryos.

To reduce the risks of immune rejection during the transplantation of tissues generated from allogeneic hiPSCs, a bank of allogeneic clinical GMP cell lines is being considered. ${ }^{12,17}$ This iPSC library will be prepared from donors homozygous for major HLA types. With regard to cartilage regeneration, the iPSC line in the library with the HLA type that matches the patient's HLA types will be selected, subjected to chondrogenic differentiation, and used for transplantation. It is estimated that a bank of 100 cell lines homozygous for major HLA types from each race would match the majority of the population. ${ }^{12,18}$ However, the preparation of dozens of clinical GMP iPSC lines is not easy, because preclinical and clinical tests are necessary for each cell line. This demand could especially be a problem when preparing iPSCs for individuals whose HLA types are rare. On the other hand, many recipients have received the transplantation of allogeneic cartilage without matching for HLA types and without the use of immunosuppressive drugs. The transplantation of particulated juvenile articular cartilage, a type of allogeneic cartilage, has given good clinical results ${ }^{5}$ so far, although the long-term clinical outcome remains to be reported. Together with previous reports, the in vitro experiments in the present study suggest that hiPS-Carts are no more antigenic than human cartilage. These findings imply that hiPS-Carts prepared from a single iPSC clone can be transplanted into patients even if the HLA types do not match. An unlimited amount of hiPSCarts can be theoretically produced from a single hiPSC clone, because hiPSCs can be expanded almost infinitely. Therefore, the use of a single hiPSC clone could reduce the cost and contribute to improve the quality of tissues for transplantation.

There are several limitations in this study. One limitation is that we compared the immunogenicity of hiPS-Cart with osteoarthritic cartilage. Comparisons with healthy cartilage derived from embryos, juveniles, or adults would contribute to further characterizing the immunogenic features of hiPSCarts, although obtaining such cartilage is not easy in certain countries. Another limitation is that the experiments in this study are in vitro only. The transplantation of allogeneic iPSC-derived cartilage in defects in the articular cartilage of an animal model is necessary.

Together with previously reported data on the immunogenicity of cartilage and chondrocytes, ${ }^{2}$ the results in the present study suggest that hiPS-Carts are no more antigenic than human cartilage. Although further studies are needed, allogeneic hiPS-Carts could be a new cell/tissue source for transplantation into articular cartilage defects in clinical settings.

\section{Acknowledgments}

The authors thank Miho Morioka, Yasuhito Yahara, Kaori Fujita, and Shin Kaneko for assistance and helpful discussions, and Peter Karagiannis for reading the article. They also thank Yudo Hachiya and Hiroki Watanabe (Hachiya Orthopaedic Hospital) for preparing the human cartilage samples. This study was supported in part by Scientific Research Grants No. 15H02561 (to N.T.) from MEXT, and Centers for Clinical Application Research on Specific Disease/Organ (to N.T.), Core Center for iPS Cell Research (to N.T.), and the Program for Intractable Diseases Research utilizing diseasespecific iPS cells (to N.T.) from AMED.

\section{Disclosure Statement}

No competing financial interests exist.

\section{References}

1. Chesterman, P.J., and Smith, A.U. Homotransplantation of articular cartilage and isolated chondrocytes. An experimental study in rabbits. J Bone Joint Surg Br 50, 184, 1968.

2. Adkisson, H.D., Milliman, C., Zhang, X., Mauch, K., Maziarz, R.T., and Streeter, P.R. Immune evasion by neocartilage- 
derived chondrocytes: implications for biologic repair of joint articular cartilage. Stem Cell Res 4, 57, 2010.

3. Arzi, B., DuRaine, G.D., Lee, C.A., Huey, D.J., Borjesson, D.L., Murphy, B.G., Hu, J.C., Baumgarth, N., and Athanasiou, K.A. Cartilage immunoprivilege depends on donor source and lesion location. Acta Biomater 23, 72, 2015.

4. Erdmann, J. ISTO technologies aims to rescue damaged joints. Chem Biol 18, 275, 2011.

5. Farr, J., Tabet, S.K., Margerrison, E., and Cole, B.J. Clinical, radiographic, and histological outcomes after cartilage repair with particulated juvenile articular cartilage: a 2-year prospective study. Am J Sports Med 42, 1417, 2014.

6. Tompkins, M. DeNovo NT allograft. Oper Tech Sports Med 21, 82, 2013.

7. Adkisson, H.D., 4th, Martin, J.A., Amendola, R.L., Milliman, C., Mauch, K.A., Katwal, A.B., Seyedin, M., Amendola, A., Streeter, P.R., and Buckwalter, J.A. The potential of human allogeneic juvenile chondrocytes for restoration of articular cartilage. Am J Sports Med 38, 1324, 2010.

8. Takahashi, K., and Yamanaka, S. Induction of pluripotent stem cells from mouse embryonic and adult fibroblast cultures by defined factors. Cell 126, 663, 2006.

9. Takahashi, K., Tanabe, K., Ohnuki, M., Narita, M., Ichisaka, T., Tomoda, K., and Yamanaka, S. Induction of pluripotent stem cells from adult human fibroblasts by defined factors. Cell 131, 861, 2007.

10. Yamashita, A., Morioka, M., Yahara, Y., Okada, M., Kobayashi, T., Kuriyama, S., Matsuda, S., and Tsumaki, N. Generation of scaffoldless hyaline cartilaginous tissue from human iPSCs. Stem Cell Reports 4, 404, 2015.

11. Tsumaki, N., Okada, M., and Yamashita, A. iPS cell technologies and cartilage regeneration. Bone 70, 48, 2015.

12. Okita, K., Matsumura, Y., Sato, Y., Okada, A., Morizane, A., Okamoto, S., Hong, H., Nakagawa, M., Tanabe, K., Tezuka, K., Shibata, T., Kunisada, T., Takahashi, M., Takahashi, J., Saji, H., and Yamanaka, S. A more efficient method to generate integration-free human iPS cells. Nat Methods 8, 409, 2011.

13. Yahara, Y., Takemori, H., Okada, M., Kosai, A., Yamashita, A., Kobayashi, T., Fujita, K., Itoh, Y., Nakamura, M.,
Fuchino, H., Kawahara, N., Fukui, N., Watanabe, A., Kimura, T., and Tsumaki, N. Pterosin B prevents chondrocyte hypertrophy and osteoarthritis in mice by inhibiting Sik3. Nat Commun 7, 10959, 2016.

14. Page, C.S., Holloway, N., Smith, H., Yacoub, M., and Rose, M.L. Alloproliferative responses of purified $\mathrm{CD} 4^{+}$and $\mathrm{CD} 8^{+}$ $\mathrm{T}$ cells to endothelial cells in the absence of contaminating accessory cells. Transplantation 57, 1628, 1994.

15. Ikeda, N., Kojima, H., Nishikawa, M., Hayashi, K., Futagami, T., Tsujino, T., Kusunoki, Y., Fujii, N., Suegami, S., Miyazaki, Y., Middleton, D., Tanaka, H., and Saji, H. Determination of HLA-A, -C, -B, -DRB1 allele and haplotype frequency in Japanese population based on family study. Tissue Antigens 85, 252, 2015.

16. Bretscher, P., and Cohn, M. A theory of self-nonself discrimination. Science 169, 1042, 1970.

17. Turner, M., Leslie, S., Martin, N.G., Peschanski, M., Rao, M., Taylor, C.J., Trounson, A., Turner, D., Yamanaka, S., and Wilmut, I. Toward the development of a global induced pluripotent stem cell library. Cell Stem Cell 13, 382, 2013.

18. Gourraud, P.-A., Gilson, L., Girard, M., and Peschanski, M. The role of human leukocyte antigen matching in the development of multiethnic "Haplobank" of induced pluripotent stem cell lines. Stem Cells 30, 180, 2012.

Address correspondence to: Noriyuki Tsumaki, MD, PhD Cell Induction and Regulation Field Department of Cell Growth and Differentiation Center for iPS Cell Research and Application Kyoto University

53 Kawahara-cho, Shogoin, Sakyo-ku Kyoto 606-8507 Japan

E-mail: ntsumaki@cira.kyoto-u.ac.jp

Received: May 12, 2016

Accepted: September 13, 2016

Online Publication Date: October 31, 2016 